\title{
DEVANT L'ALIENATION, LA CONSCIENCE DE SOI À TRAVERS LE LANGAGE POETIQUE D'AGOSTINHO NETO, JOSÉ CRAVEIRINHA ET NOÉMIA DE SOUSA
}

\author{
DIANTE DA ALIENAÇÃO, A CONSCIÊNCIA DE SI ATRAVÉS DA LINGUAGEM POÉTICA DE \\ AGOSTINHO NETO, JOSÉ CRAVEIRINHA E NOÉMIA DE SOUSA
}

\begin{abstract}
Alexandre Coly ${ }^{1}$
${ }^{1}$ Université Assane Seck de Ziguinchor (UASZ), Ziguinchor, Casamansa, Sénégal a.coly@univ-zig.sn
\end{abstract}

Recebido em: 25 abr. 2018

Aceito em: 29 mai. 2018

\begin{abstract}
Resumo: O gozo da consciência de si diante da dominação arbitrária e da alienação colonial é um das forças da linguagem poética de Agostinho Neto, Noémia de Sousa e José Craveirinha. Perante a situação colonial complexa, esses autores da África lusófona desenvolveram níveis de resistência tanto na escrita, como nas armas para derrubar a lógica da ordem estabelecida colonialista. Mas, neste artigo, vamos nos interessar muito mais pelo papel da linguagem poética como uma forma de libertação das consciências cegadas pelo pensamento dominante, isto é, o do colonizador. Por isso, entendemos que o ser angolano, moçambicano e africano encontra-se no centro de seus discursos poéticos, que reafirmam suas particularidades de maneira significativa. Nesta perspectiva, a vontade de ser independente enquanto ser humano se tornou uma das exigências do colonizado, assim, esses poetas retornam ao léxico local, buscando enraizar-se na terra e contestar a desumanidade desta ordem colonial. Com tantas mazelas no ar, era preciso provocar através da poesia uma sensibilidade singular sobre esta situação caótica dos povos africanos. Portanto, a angolanidade, a moçambicanidade e africanidade são linguisticamente invocadas para defender suas heranças socio-culturais e denunciar as violações praticadas pelo colonizador.
\end{abstract}

Palavras-chave: Linguagem. Alienação. Consciência de si. Africanidade.

Résumé: La jouissance de la conscience de soi face à la domination arbitraire et à l'aliénation coloniale est une des forces du langage poétique d'Agostinho Neto, Néomia de Sousa et José Craveirinha. Devant la complexité de la situation coloniale, ces poètes d'Afrique lusophone ont développé des niveaux de résistance tant sur le plan de l'écriture que sur la lutte armée, pour faire avorter la logique de l'ordre établi colonialiste. Nous allons nous focaliser sur le langage poétique comme une forme de libération des consciences aveuglées par la pensée dominante, c'est-à-dire celle du colonisateur. En cela, nous comprenons que l'être angolais, mozambicain et africain se trouve au centre de leurs discours poétiques, qui réaffirment leurs particularités de manière significative. Dans ce cadre, la volonté d'être indépendant en tant qu'être humain est devenue une des exigences du colonisé, que ces poètes ont assumés le langage poétique du retour au lexique local et de condamner la déshumanité exercée par cet ordre établi. Avec autant de blessures qui planent, il fallait provoquer à travers la poésie une sensibilité singulière portée sur la situation chaotique des peuples africains. Par conséquent, l'angolanité, la mozambicanité et l'africanité sont linguistiquement invoquées dans le but de défendre leurs héritages socio-culturels et dénoncer les violations pratiquées par le colonisateur portugais.

Mots-clé: Langage. Aliénation. Conscience de soi. Africanité.

\section{Introdução}


Être dans une situation évidente d'avoir été chosifié et d'y prendre conscience de manière intrinsèque est sans doute une des marques rayonnantes du langage poétique de l'appel du soi collectif chez les poètes luso-africains - José Craveirinha, Noémia de Sousa et Agostinho Neto. En effet, la complexité de la situation coloniale en Afrique lusophone (de 1960 à 1975) a suscité chez ces poètes une remise en question de la dictature coloniale Salazariste pour permettre à leurs peuples de vivre la liberté en tant qu'être africain et se de libérer du joug de l'aliénation coloniale. C'est donc par une exigence douloureuse de soi que le contenu et le style de leurs poèmes révèlent la nécessité de se prendre en main. Cela se constatera à travers l'analyse de chaque poème afin de mieux comprendre le langage poétique comme un vecteur d'émancipation de soi vis-à-vis de l'aliénation.

\section{Le langage poétique comme nécessité d'être soi-même}

Si la vraie violence coloniale est consubstantielle à l'aliénation du colonisé, ou encore à l'effacement de son identité, le poète par son verbe devient une des colonnes vertébrales pour engendrer un véritable retour de soi. En cela, le poème «Sangue da minha mãe », du poète mozambicain José Craveirinha, témoigne de ce caractère de la nécessité d'être soi-même :

\begin{tabular}{|c|c|}
\hline Sangue da Minha mãe & Sang de ma mère \\
Xipalapala1 está chamar & C'est l'appelle du cor \\
oh, sangue da minha Mãe & xigubo² vai começar \\
xigubo vai rebentar & oh, la danse guerrière va commencer \\
e xipalapala está chamar sangue & la danse guerrière va commencer et appelle \\
de minha Mãe. & les fils de ma patrie \\
Oh, sangue de minha Mãe & Oh, sang de ma patrie \\
xigubo está chamar & C'est la danse guerrière \\
xigubo está chamar & C'est l'appelle du cor \\
\hline
\end{tabular}

\footnotetext{
${ }^{1}$ Trompette faite avec la corne de l'antilope et qui sert à lancer un appel ou à convoquer le peuple pour une réunion (CRAVEIRINHA, 1990, p. 228).

${ }^{2}$ Danse d'exaltation des guerriers. Elle se pratique ou après le combat (Idem).
}

Pensares em Revista, São Gonçalo-RJ, n. 12, p. 160-179, 2018 


\begin{tabular}{|c|c|}
\hline $\begin{array}{l}\text { e eu vou entrar no xigubo } \\
\text { sangue de minha Mãe! } \\
(. . .) \\
\text { Xipalapala está chamar } \\
\text { Culucumba de minha Mãe está } \\
\text { rezar mato vai acordar } \\
\text { xigubo vai começar } \\
\text { oh... sangue de minha Mãe } \\
\text { xigubo vai começar } \\
\text { e xipalapala vai cruzar os } \\
\text { caminhos do rio e do mar } \\
\text { gritar e suar no xigubo } \\
\text { gritar sangue de minha Mãe! }\end{array}$ & $\begin{array}{c}(\ldots) \\
\text { C'est l'appelle du rassemblement de ma } \\
\text { patrie les dieux de ma patrie prient } \\
\text { la forêt va se réveiller } \\
\text { la danse guerrière va commencer } \\
\text { oh... sang de ma patrie } \\
\text { la danse guerrière va commencer et le son du } \\
\text { cor va répandre sur les chemins du fleuve, de } \\
\text { la mer } \\
\text { C'est la danse animée de guerriers pour } \\
\text { défendre la patrie! }\end{array}$ \\
\hline
\end{tabular}

CRAVEIRINHA, 1990, p. 228. Tradução nossa.

Avec ce poème, Craveirinha pose un acte de conscience de soi authentique. En effet, le titre du poème illustre de façon remarquable l'architecture d'une construction identitaire dans un contexte colonial. Le poète définit sans faille le sens de l'être mozambicain face aux injonctions arbitraires de l'ordre établi. L'évocation intentionnelle de sa terre natale suggère son attachement à ses origines et sa culture. Cela revient à dire que le poète manifeste son lien tellurique avec sa terre natale. D'autre part, il y a un désir de la part du poète de magnifier et d'exalter son héritage culturel négro-africain au tour du «xipalapala » et du «xigubo ». L'exaltation abondante des instruments traditionnels traduit le monde dans lequel il a vécu. En d'autres termes, le « xipalapala » et le «xigubo » rendent possible un dialogue entre le visible et l'invisible. Ce sont également des témoins de la danse ou des acteurs de la tradition orale mozambicaine et négro-africaine. C'est ce legs ancestral autour de la parole qui anime l'esprit du poète en quête d'un devenir. Dans cette perspective, le poète exhorte les guerriers à défendre la patrie en danger et d'être de véritables remparts contre la fabrique de l'aliénation complexité coloniale.

Le « xipalapala » et le « xigubo » sont comme une source de vie dans l'esprit du poète. De même, leurs forces d'appel restent sans doute un moyen de comprendre à la fois l'être mozambicain et la société mozambicaine, "Xipalapala vous appelle», "xigubo va commencer ». C'est en outre une manière d'être soi-même. C'est pourquoi le poète met en filigrane dans ce poème des mots de sa langue maternelle (le ronga) «xipalapala », "xigubo », pour en indiquer parfois l'affirmation de l'identité 
nationale et son origine culturelle. Par ce biais, Craveirinha met en relief sa négritude. II manifeste donc son africanité, son appartenance à une culture africaine en même temps qu'il veut sauver sa terre africaine confisquée par l'ordre colonial. Craveirinha ne se limite pas à évoquer ces instruments musicaux, il se réclame et s'approprie ce legs ancestral travesti par la pensée dominante de l'époque. Ce qui fait du poète un jalon de conscience vis-à-vis de son peuple et, sur de l'homme.

Cela renvoie à un langage poétique d'auto-affirmation assez prononcée et de mise en valeur des caractéristiques du champ littéraire négro-africain chez Craveirinha. D’après Patrick Chabal,

[...] en exprimant les vertus des cultures négro-africaines, Craveirinha a réussi à transformer l'apparente infériorité culturelle des peuples colonisés et subjugués, en les réhabilitant en créativité, force et espoir. Donc, Craveirinha a contribué par sa poésie à l'invention d'une littérature autonome et moderne. (CHABAL, 1995, p. 56. Tradução nossa.) ${ }^{3}$

Chabal souligne l'importance de la voix de Craveirinha dans le champ littéraire négro-africain, notamment au Mozambique. Pour lui, le poète mozambicain reste une figure remarquable, dans la mesure où il parvient par le biais de sa poésie à redonner une nouvelle vie aux cultures négro-africaines considérées comme inférieures par l'Occident. C'est la raison pour laquelle le poème, «Sang de ma patrie », réaffirme l'aspiration du poète à réhabiliter son identité culturelle en employant un lexique local. C'est-à-dire le vocabulaire de sa langue maternelle, le ronga. Tout au long du poème, le poète manifeste son attachement à sa langue maternelle afin d'exhiber sa mozambicanité.

Dans le même ordre d'idées, la référence à la figure maternelle comme source d'identité se prolonge dans un autre poème, « Mãe »,

\begin{tabular}{|l|l|}
\hline Mãe & $\begin{array}{l}\text { Ma mère } \\
\text { Minha Mãe: }\end{array}$ \\
& $\begin{array}{l}\text { Maman: } \\
\text { Je porte dans mes veines }\end{array}$ \\
\hline
\end{tabular}

\footnotetext{
${ }^{3}$ Dans le texte original : "Ao expressar as virtudes das culturas africanas, Craveirinha tentou transformar a aparente inferioridade cultural dos povos colonizados e subjugados, num resgate de criatividade, força e esperança. Portanto, este autor "contribuiu com sua poesia para a "invenção" de uma literatura autônoma e moderna."
}

Pensares em Revista, São Gonçalo-RJ, n. 12, p. 160-179, 2018 


\begin{tabular}{|l|l|}
\hline Trago a resina das velhas & la sève des vieux arbres de la \\
árvores & forêt \\
da floresta nas minhas veias & Et le destin de naissance au \\
E a sina de nascença & milieu des danses autour du \\
no meio das baladas à volta & feu \\
da fogueira & tu sais pour toujours que c'est \\
tu sabes como é sempre uma & une nouvelle douleur tu sais \\
dor nova & ou le sais-tu ou non, \\
sabes ou não sabes, minha & Maman ? \\
Mãe? & Et dans la saveur de \\
E no sabor do encantamento, & l'enchantement, Maman de \\
Mãe dos nossos & nos fétiches présents \\
desenfeitiçados feitiços & ancestraux l'exorcisme naïf \\
ancestrais & de tes perles \\
o exorcismo ingénuo das tuas & le merveilleux maheu de tes \\
missangas & chansons et le secret de ton \\
o maravilhoso maheu das & corps possédé mais de sang \\
tuas canções e segredo do & maternel inviolable d'où mon \\
teu corpo possuído mas & destin est né. \\
materno sangue inviolàvel & \\
donde minha sina nasceu. & \\
\hline
\end{tabular}

CRAVEIRINHA, p. 95. Tradução nossa.

Le langage poétique à tout son sens dans le combat contre la machine colonialiste. Dans ce cadre, la question de l'identité culturelle et de la conscience de soi résonnent à travers l'éloge du sang de sa mère - son totem identitaire. Sémantiquement, ce poème exprime une tendresse maternelle ressentie par la voix poétique et une sensibilité visant à éterniser la figure maternelle. Ce qui correspond à une forme d'identification au sang maternel. Par ce biais, Craveirinha s'adonne à exalter tout ce qui nourrit culturellement son univers maternel pour sentir qu'il existe, « les fétiches présents ancestraux », «les vieux arbres » et « le sang maternel inviolable ». Le poète montre de façon subtile qu'il s'identifie à son milieu naturel, à sa terremère, le Mozambique, ou encore à sa mère africaine. Craveirinha vient incarner par l'imagination l'apport de l'identité culturelle mozambicaine et négro-africaine dans son acte poétique. II s'inscrit dans la quête d'une identité et d'une d'affirmation de ses origines à travers son acte poétique assez original. C'est la raison pour laquelle le faire poétique de Craveirinha est en phase avec l'essence qui fonde son être, c'est-à-dire,

\footnotetext{
${ }^{4}$ Maheu est une boisson traditionnelle très nutritive, faite à base de riz et de farine de blé, mélangée avec du sucre. Tradução nossa.
}

Pensares em Revista, São Gonçalo-RJ, n. 12, p. 160-179, 2018 
se reconnaître en tant que fils de la terre mozambicaine. Par son langage poétique qui fait un retour réel aux valeurs culturelles de son peuple sonne son attachement inconditionnel à la terre mère « sans maternel inviolable ». Selon les propos d'Ana Malfada Leite,

De cette manière, l'origine culturelle du poète va se révéler à travers son acte poétique, où se croisent bien ajustées plusieurs options esthétiques ajustées s'imbriquent. Ce fait nous amène à faire la présentation de l'auteur en prenant en compte des données biographiques et des énoncés qui tendent à se construire en biographèmes (révélant la représentation que le poète fait de soimême et de ses origines dans les poèmes Karingana ua Karingana), ce qui nous permet de mettre en filigrane l'important rôle thématique de l'origine familiale, géographique, culturelle et linguistique de l'auteur. (LEITE, 1991, p. 17. Tradução nossa.) ${ }^{5}$

Ana Malfada Leite montre les caractéristiques de la poésie de José Craveirinha. Pour elle, l'ouvrage Karingana ua Karingana représente le milieu traditionnel mozambicain dont s'inspire le poète pour retrouver ses racines et prendre ainsi conscience de ses origines. C'est pourquoi elle insiste sur la forte influence du facteur linguistique local, ses liens maternels et sur l'espace géographique dans la poésie de Craveirinha. Cela permet de dire que le poète veut s'émanciper, une sorte de fondation de soi-même autour des paroles poétiques évoquant son mode d'être. Revenant au poème, "Ma mère », on note que la structure linguistique du poème intensifie l'importance de la symbolique du sang maternel, pour témoigner de l'inséparable valeur maternelle. Cette intensification est une force qui se manifeste dans l'évocation de la mère.

D'un autre côté, il convient de signaler que le poète ne rejette pas pour autant ses origines paternelles. II est partagé entre l'amour maternel et l'admiration pour son père. Grâce à eux il est devenu mozambicain. II éprouve une profonde fierté :

\footnotetext{
${ }^{5}$ Dans le texte original: "A origem cultural do poeta vai, deste modo, revelar-se no seu fazer poético, em que se cruzam, ajustadas, várias opções estéticas. Este facto leva-nos a fazer a apresentação do autor tomando em consideração dados biográficos e enunciados que tendem a construir-se em biografemas (revelando a representação que o poeta faz de si próprio e de suas raízes nos poemas Karingana ua Karingana), o que nos permite destacar o importante papel temático da origem familiar, geográfica, cultural e linguística do autor."
}

Pensares em Revista, São Gonçalo-RJ, n. 12, p. 160-179, 2018

DOI:10.12957/pr.2018.33957 


\begin{tabular}{|c|c|}
\hline $\begin{array}{l}\text { Ao Meu Belo Pai Ex- } \\
\text { emigrante } \\
\text { Pai: as maternas palavras } \\
\text { de signos vivem e revivem } \\
\text { no meu sangue e } \\
\text { pacientes esperam ainda a } \\
\text { época de colheita } \\
\text { enquanto soltas já são as } \\
\text { tuas sentimentais } \\
\text { sementes de emigrante } \\
\text { português [...] E na minha } \\
\text { rude e grata sinceridade } \\
\text { não esqueço } \\
\text { meu antigo português puro } \\
\text { que me geraste no ventre } \\
\text { de uma tombasana } \\
\text { eu mais um novo } \\
\text { moçambicano semiclaro } \\
\text { para não ser igual a um } \\
\text { branco qualquer e } \\
\text { seminegro para jamais } \\
\text { renegar um glóbulo que } \\
\text { seja dos Zambezes do } \\
\text { meu sangue. }\end{array}$ & $\begin{array}{l}\text { A mon bien aimé Père } \\
\text { Ex-émigré } \\
\text { Père: les paroles douces } \\
\text { et maternelles bercent et } \\
\text { berceront toujours mon } \\
\text { âme et feront de moi un } \\
\text { homme accompli, tandis } \\
\text { que l'éducation } \\
\text { occidentale que j'ai reçue } \\
\text { à tes côtés fait déjà ses } \\
\text { effets en moi [...] } \\
\text { Je suis de nature rude } \\
\text { mais je vous en sais } \\
\text { je n'oublie pas mon vieux } \\
\text { portugais pur qui m'a } \\
\text { procrée le ventre d'une } \\
\text { tombosana } \\
\text { Je ne suis pas totalement } \\
\text { un mozambicain à peau } \\
\text { blanche pour l'égal d'un } \\
\text { blanc, je ne suis pas non } \\
\text { plus en plus un } \\
\text { mozambicain à peau noire } \\
\text { pour être l'égal d'un noir } \\
\text { Ce qui fait que je ne } \\
\text { pourrais jamais renier mon } \\
\text { sang. }\end{array}$ \\
\hline
\end{tabular}

CRAVEIRINHA, 1990, p. 108. Tradução nossa.

Dans le poème " $A$ mon bien aimé Père Ex-émigré », Craveirinha exprime particulièrement son héritage culturel double : portugais et mozambicain. II assume son identité métissée, « je ne suis pas totalement blanc pour être considéré comme un blanc et je ne suis pas totalement noir pour être considéré comme un noir. ». En d'autres termes, il s'affirme autour de l'union de sa mère mozambicaine et de son père portugais. Durant un entretien avec Michel Laban, Craveirinha déclarait qu'il était né deux fois : " la première fois fut le jour de sa naissance et la deuxième, le jour où il a pris conscience qu'il était métisse ".6 (LABAN, 1998, vol.2, p.10-13 Tradução nossa).

De même, il affirmait la thèse suivante: « je ne suis pas divisé, je suis entre deux cultures. On ne m'a pas scindé en deux. J'aime les deux cultures». (CRAVEIRINHA,

${ }^{6}$ Dans le texte original: "a primeira no dia do parto e a segunda no dia em que tomou consciência de que era mestiço."

Pensares em Revista, São Gonçalo-RJ, n. 12, p. 160-179, 2018 
1990, p. 54. Tradução nossa ${ }^{7}$ ) Ces deux affirmations indiquent dans une certaine mesure l'orientation poétique de Craveirinha à travers le poème ci-dessus.

Le discours poétique suggère que le sujet parlant se souvient des affections intenses de la culture maternelle, "Père: les douces paroles de ma mère bercent et berceront toujours dans mon âme et feront de moi un homme accompli ». II manifeste son désir d'affirmation en assumant cette double appartenance. II convient de dire que les caractéristiques de son discours poétique renvoient à la tradition orale négroafricaine à l'image du titre de l'ouvrage, Karingana ua Karingana - il était une fois dans la langue mozambicaine Ronga. La valeur intensive de la présence de l'oralité dans le poème est une manière de marquer ses distances vis-à-vis du modèle occidental. Dans l'esprit socio-culturel négro-africain, l'oralité est consubstantielle à la mémoire, parce que étant le chemin du retour de soi-même, pour se désaliéner du cordon colonial portugais. Le caractère typiquement oral du poème est un référent linguistique de son royaume d'enfance. C'est dans ce sens que Craveirinha évoque sa mozambicanité en mettant en valeur une prise de conscience de soi et de son africanité.

Dans un autre poème qui illustre sa vision, Craveirinha intensifie son enracinement à la terre mozambicaine ainsi que son attachement au monde négroafricain:

\begin{tabular}{|l|l|}
\hline Manifesto & Manifeste \\
[...] Oh! E meu peito da & {$[\ldots]$ Oh! Et mon corps le plus } \\
tonalidade mais bela do breu & beau et notre baobab notre \\
e no embondeiro da nossa & espoir sans égal marqué par \\
inaudita esperança gravado o & le totem le plus invincible du \\
tótem mais invencível tótem & Monde et ma voix affective \\
do Mundo e minha voz & d'homme du Tanganyika, du \\
estentória de homem do & Congo, d'Angola, du \\
Tanganhica do Congo, & Mozambique et du Sénégal. \\
Angola, Moçambique e & Ah! Encore une fois \\
Senegal. & moi le chef zulu \\
Ah! Outra vez eu chefe zulo & Moi le chasseur bantu \\
eu azagaia banto & Moi le lanceur de mauvais \\
& sort contre les insatiables \\
\hline
\end{tabular}

7 Dans le texte original: "Não estou dividido, estou repartido. Não me dividiram. Eu amo as duas culturas."

Pensares em Revista, São Gonçalo-RJ, n. 12, p. 160-179, 2018

DOI:10.12957/pr.2018.33957 


\begin{tabular}{|l|l|}
\hline eu lançador de malefícios & criquets de malheur \\
contra as insaciáveis pragas & envahisseurs \\
de gafanhotos invasores & Moi le tambour \\
Eu tambor, & Moi surama \\
Eu suruma & Moi le noir suaili \\
Eu negro suaili & Moi le Tchaca \\
Eu Tchaca & Moi le Mahazul et le \\
Eu Mahazul e Dingana. & Dingana. \\
\hline
\end{tabular}

CRAVEIRINHA, 1980, p. 33

En s'enracinant dans sa terre-mère, le Mozambique, Craveirinha vit son identité d'un côté, et, de l'autre, il décrit des images suggérant la virilité de l'homme noir : « Et mon corps le plus beau». La valeur sémantique du «baobab » correspond à une force naturelle où le poète puise son énergie pour mieux s'identifier à la nature mozambicaine généreuse. Le " baobab " et le «totem " sont ici des signes, des éléments qui symbolisent la vitalité de l'univers culturel mozambicain et sont les témoins d'un cadre de dialogue fraternel. Par l'évocation sans réserve aucune du totem, Craveirinha s'enracine dans ses croyances ancestrales et dans son identité, "le totem plus invincible du Monde ». C'est son être qui entre en communion avec le cadre identitaire mozambicain.

Ce qui frappe, par ailleurs, c'est la façon dont Craveirinha s'imbrique avec d'autres voix africaines pour exalter tout un continent. Dans l'avant dernière strophe, il met en valeur la diversité culturelle mozambicaine. Grâce à ce poème, le poète évoque de manière polyphonique l'image des cultures africaines durant la répression coloniale. II exalte les forces vivantes de l'Afrique et dénonce l'occupation étrangère en même temps : "Ah! Encore une fois moi le chef Zulu, moi le chasseur Bantu moi le lanceur de mauvais sort contre les insatiables criquets de malheur envahisseurs». La valeur anaphorique du « moi » dans ce poème suggère une personnalité multiple et annonce plusieurs fonctions en même temps. Mais tout ramène vers l'Afrique et l'homme noir, " ma voix affective d'homme du Tanganyika, du Congo». Ainsi, les images de la dernière strophe montrent le caractère oral du poème. Cela signifie que Craveirinha fait intervenir le langage oral dans ses écrits poétiques, «moi le tambour », « moi le noir suaili », « moi le Tchaca ». Par ce biais, la multiplication du moi montre 
que le poète exprime la symbolique de l'africanité et de la Négritude. II réhabilité l'image de l'homme noir et de l'africain.

\section{L'exigence de se sentir mozambicain et africain}

Après Craveirinha, nous allons analyser maintenant le poème, « La femme qui rit de la vie et de la mort», de la poétesse mozambicaine Noémia de Sousa, qui aborde la question de la conscience de soi et l'africanité dans une perspective un peu différente:

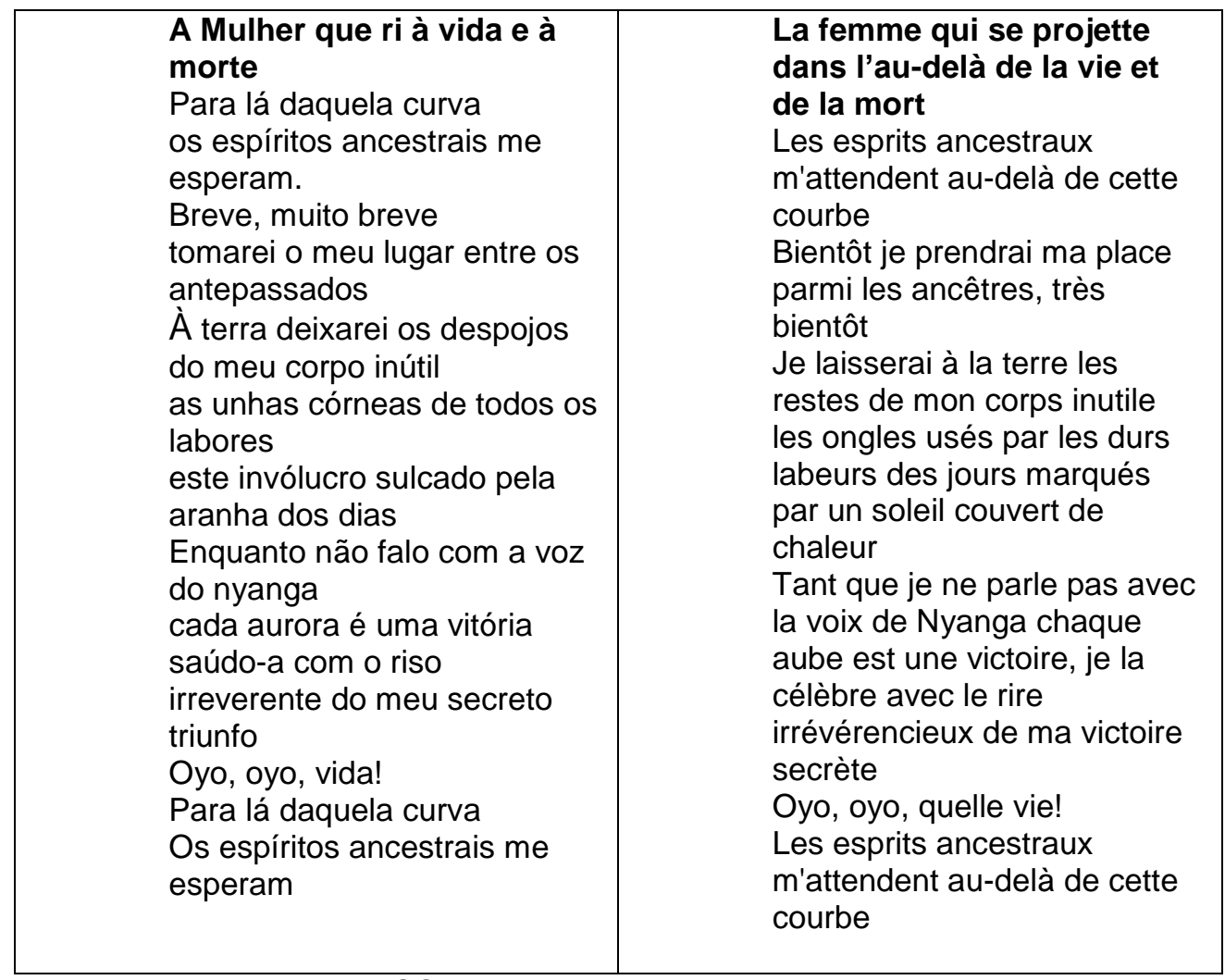

SOUZA, 1988, p. 149. Tradução nossa.

Noémia de Sousa est une pionnière de la littérature mozambicaine. Dans ce poème, elle exprime les marques de son identité par la prise de conscience de soi. Dès la première strophe, la voix poétique dialogue entre l'au-delà et le monde des vivants, «Les esprits ancestraux m'attendent au-delà de cette courbe ». A ce titre, elle Pensares em Revista, São Gonçalo-RJ, n. 12, p. 160-179, 2018 
évoque un mode d'être à la manière africaine (les morts ne sont pas morts). En outre, elle utilise des mots qui suggèrent les signes de sa mozambicanité, c'est-à-dire de sa culture étant donné que la culture est un élément constitutif de l'identité de l'individu. Elle donne une autre fonction primordiale à la tradition ancestrale mozambicaine et africaine, « les esprits ancestraux m'attendent », « Bientôt je prendrai place parmi les ancêtres ». C'est l'appel de la symbiose du corps et de l'esprit

La deuxième strophe indique la volonté de la voix poétique de se fondre dans ce monde traditionnel pour susciter un retour véritable à ses ancêtres. Elle exprime son appartenance au monde noir, donc à la Négritude. Ce monde a véritablement un sens, «Bientôt je prendrai ma place parmi les anciens. ». Noémia de Sousa tend radicalement vers la plénitude du monde traditionnel mozambicain afin de mieux sentir son existence. Elle nous révèle symboliquement le monde animiste africain.

Chez Noémia de Sousa, il est question de repenser la place des valeurs culturelles mozambicaines et les vivre sans aucune réserve. C'est dans une certaine mesure mettre en valeur les éléments culturels de son pays. Cela s'explique par la densité des images de la tradition culturelle mozambicaine dans la troisième strophe. Une telle démarche donne lieu à une dynamique identitaire. D'après les propos de Serra,

Noémia de Sousa est un cas unique d'expression explosive de son identité africaine, sa voix surprend principalement pour cette raison. Sa poésie est aussitôt envahie par des voix, elle est la voix des sans voix, elle incarne les personnages submergés dans le quotidien qui leur refuse l'existence, pour ne pas parler d'identité. (SERRA, 1998, p. 90. Tradução nossa). ${ }^{8}$

Serra montre à travers cette déclaration l'importance de la poésie de Noémia de Sousa. En effet, Serra considère qu'elle est le symbole en devenir du combat pour la réhabilitation de son identité aliénée. II soutient que sa poésie reste un haut lieu, «d'expression explosive», c'est-à-dire de conscience de soi et d'une prise en charge

\footnotetext{
8 Dans le texte original: "Noémia de Sousa é caso único de explosão identitária, a sua voz surpreende justamente por esta razão. A sua poesia é logo invadida por vozes, ela é a voz dos que não a têm, ela incarna as personagens submersas no quotidiano que lhes recusa a existência, para não falar de identidade."
}

Pensares em Revista, São Gonçalo-RJ, n. 12, p. 160-179, 2018 
des «sans voix ». Cette pensée de revendication identitaire de Serra peut être appliquée au poème que nous analysons ci-dessous où Noémia de Sousa s'adonne à une sorte d'obsession, intégrant même les aspects animistes.

La structure du poème « la femme qui se projette dans l'au-delà de la vie et de la mort » nous donne une idée de l'architecture socioculturelle mozambicaine. Noémia de Sousa vient témoigner à travers ce poème de l'importance des valeurs culturelles africaines ainsi que la place de la femme dans la construction permanente de l'identité nationale. A cet effet, le titre du poème est assez suggestif dans la mesure où il résume la force intérieure de la femme - elle ne craint rien, ni la réalité de la vie, ni la mort. En ce sens, la voix poétique exprime par là le caractère éphémère du corps humain, « Je laisserai à la terre mon corps inutile. ». C'est justifier et renforcer son désir de dialoguer avec les ancêtres invisibles pour se sentir existée.

Par ailleurs, Noémia de Sousa n'a pas besoin d'intermédiaire. Elle communique directement avec les ancêtres, «Tant que je ne parle pas avec la voix nyanga, chaque aube est une victoire... ». Ceci lui donne le même pouvoir que le sorcier. Ce combat est secret et se fait en dehors des canaux rituels, d'où l'autosatisfaction. C'est en outre une nécessité de manifester l'identité culturelle mozambicaine face à l'aliénation culturelle imposée par le système colonial portugais. Cette manifestation par un langage identitaire s'articule autour de la voix poétique, dans la mesure où elle représente aussi la femme mozambicaine et africaine. Selon les propos de Momplè Lília, « [...] la femme mozambicaine est le principal canal de diffusion de valeurs culturelles, de traditions et rites comme, par exemple, l'esprit de solidarité, d'hospitalité, de vénération des plus vieux, les rites de naissance, l'initiation, la réconciliation et la mort ». (In: FERRO, 1999, p.31.Tradução nossa). ${ }^{9}$

Momplè explique de façon claire que la femme mozambicaine est la colonne vertébrale de l'identité culturelle de son pays. Autrement dit, elle reste un être actif, puisqu'elle fait l'éloge des valeurs culturelles de son peuple à l'instar de Noémia de

\footnotetext{
${ }^{9}$ Dans le texte original: "[...] a mulher moçambicana é a principal difusora e transmissora de valores culturais, tradições e ritos como, por exemplo, o espírito da solidariedade e entreajuda, a hospitalidade, a veneração pelos mais velhos, os ritos de nascimento, iniciação, reconciliação e morte."
}

Pensares em Revista, São Gonçalo-RJ, n. 12, p. 160-179, 2018 
Sousa. De ce point de vue, elle est comme un modèle du monde culturel mozambicain. Le poème «La femme qui se projette de l'au-delà de la vie et de la mort » illustre bien le rôle de la femme et son apport dans la construction du processus identitaire mozambicain. Aussi, il exprime le pouvoir de la femme de se projeter vers l'au-delà et de rejoindre les ancêtres protecteurs. En un mot, Noémia de Sousa parvient à transcender les contradictions de la vie. C'est sa manière de vaincre les affres du colonialisme et ce qui lui procure une victoire sécrète. Par conséquent, sa démarche traduit une lucidité d'interrogation sur soi.

On note que le poème exprime les caractéristiques de la tradition orale. C'est-àdire que Noémia conte son univers traditionnel de manière poétique. C'est une façon de raffermir l'importance de la mémoire et de la parole entre les vivants et les morts. Cela suggère son mode d'être. En un mot, Noémia de Sousa se réapproprie de l'héritage traditionnel pour exalter à travers des images poétiques de l'identité culturelle mozambicaine et sa négritude. D'après Amadou Hampaté Bâ,

Dans les civilisations orales, la parole engage l'homme, la parole EST I'homme. D'où le respect profond des récits traditionnels légués par le passé, dont il est permis d'embellir la forme ou la tournure poétique, mais dont la trame reste immuable à travers des siècles, véhiculée par une mémoire prodigieuse qui est la caractéristique même des peuples à tradition orale. (HAMPATÉ, 2008, p. 25)

Hampaté Bâ met en relief dans ce fragment l'importance de l'oralité dans les civilisations africaines. II soutient que la parole est l'homme. Autrement dit, elle est une arme puissante agissant sur le monde africain. II s'agit là d'une prise de conscience de la valeur "[des] récits traditionnels », du fait qu'ils permettent de donner une continuité ou de transmettre aux nouvelles générations un patrimoine culturel et historique riche. C'est pourquoi Amadou Hampaté Bâ considère que les traditions orales africaines restent des mémoires indélébiles - une autre façon de véhiculer des messages, de récréer un monde du savoir être africain. Ce qui laisse croire que la parole dans les cultures africaines contient une teneur sacrée comme l'indique le poème, "La femme qui se projette de l'au-delà de la vie et de la mort ". Dans ce poème, Noémia de Sousa s'inspire poétiquement des récits traditionnels pour conter

Pensares em Revista, São Gonçalo-RJ, n. 12, p. 160-179, 2018 
et accéder au monde des vivants et des morts « Les esprits ancestraux m'attendent au-delà de cette courbe». II y a là une valeur sémantique significative par la création de l'univers traditionnel mozambicain où l'oralité et la mémoire sont une source de vie abondante. Noémia de Sousa met ainsi en valeurs ces deux éléments traditionnels pour lancer un message, c'est-à-dire la jouissance radicale de l'être soi-même face à la barbarie coloniale. C'est une manière de démystifier la culture occidentale et de marquer son africanité.

Dans un autre poème intitulé, «Sang noir », Noémia de Sousa nous replonge par son imagination dans ses racines africaines:

\begin{tabular}{|l|l|}
\hline Sangue negro & Sang noir \\
Ó minha África misteriosa e & Ô mon Afrique naturelle et \\
natural, minha virgem & mystérieuse, ma vierge violée, \\
violentada, & ma Mère \\
minha Mãe & Ô ma Maman Afrique, ngoma \\
Ó minha Mãe África, ngoma & païenne esclave sensuelle, \\
pagã, & sortilège, mystique - pardonne! \\
escrava sensual, & Que la force de ta sève touche \\
mística, sortílega - perdoa [...]! & tout! \\
Que a força da tua seiva vence & pour que je vibre \\
tudo! & pour que je crie \\
para que eu vibrasse & pour que je sente, \\
para que eu gritasse, & profondément, dans mon cœur, \\
para que eu sentisse, funda, no & ta voix, Mère! \\
sangue, a tua voz, Mãe! & Et déçue par la vie, il faut que je \\
E vencida, reconhecesse os & reconnaisse nos liens \\
nossos elos... & consanguins... et je retourne à \\
e regressasse à minha origem & mon origine millénaire \\
milenar. & \\
Mãe, minha Mãe África & Mère, ma Mère Afrique des \\
das canções escravas ao luar, & chants des esclaves au clair de \\
não posso, não posso repudiar & la lune, je ne peux, je ne peux \\
o sangue negro, o sangue & renier le sang noir, le sang mêlé \\
bárbaro que me legaste... & que tu m'as légué... \\
Porque em mim, em minha & Car en moi, dans mon âme, \\
alma, em meus nervos, & dans mes nerfs, il est plus fort \\
ele é mais forte que tudo, & que tout, il me faut vivre, il est \\
eu vivo, eu sofro, eu rio através & l'origine de ma souffrance, de \\
dele, Mãe! & mon bonheur, Mère! \\
\hline
\end{tabular}

SOUZA, 1988, p. 140-141. Tradução nossa.

Dans ce poème, Noémia de Sousa réussit à lier sa poésie avec les figures symboliques de l'histoire africaine. Au premier abord, le titre du poème est suggestif, 
«Sang noir». II renvoie à la sémantique et à la symbolique du sang des noirs où la poétesse est en fusion de manière consanguine et imaginaire. Ce qui frappe à la première strophe, c'est la métaphore de l'histoire coloniale indiquant subtilement une Afrique, « violée », « assujettie » par les abus du régime colonialiste portugais. Dans ce cadre, l'énonciation poétique de Noémia Sousa fait resurgir les traits de la Négritude. Nous voulons indiquer que les chantres de Négritude ont exprimé tour à tour leur appartenance identitaire à la Mère-Afrique et dénonçaient les pratiques inhumaines de la colonisation occidentale en terre africaine. Cela peut être assimilé aux propos de Manuel Ferreira concernant la poésie de Noémia de Sousa : «Poésie d'impact social assez fort, dénonciatrice, son langage, à bien des égards convoque l'antillais Aimé Césaire, non seulement du point de vue thématique, mais aussi par rapport au recours stylistique de résonance verbale». (FERREIRA, 1977, p.73. ${ }^{10}$ Tradução nossa)

Chez Noémia de Sousa, l'idée de conscience de soi part aussi d'un langage poétique du retour aux sources, « je retourne à mon origine millénaire ». La voix lyrique assume de manière cardinale sa particularité. En ce sens, s'opère un renouvellement de soi afin de créer une nouvelle condition de la vie en elle. Un tel désir traduit une sorte d'émancipation intérieure ainsi que celle de son peuple. La quête d'une émancipation de soi est constatée au moment où la voix poétique déclare, « pour que je vibre », « pour que je crie », « pour je sente ». Consciente de son lien avec sa « Mère Afrique » occupée et meurtrie, Noémia de Sousa se projette pour mieux sentir son Afrique. C'est en outre un dialogue filial qui se tisse entre elle et la Mère-Afrique.

Étant métisse, la Noémia de Sousa ne cesse de mettre en valeur son héritage africain, « je ne peux, je ne peux renier le sang noir ». C'est également une façon d'être soi-même, de s'affirmer en résistant aux agressions culturelles. En cela, le titre du poème rappelle celui de l'ouvrage en question, Sang noir, pour en indiquer son attachement presque viscéral à l'univers négro-africain, «Car en moi, dans mon âme,

\footnotetext{
10 Dans le texte original: "[...] poesia de forte impacto social, acusatória, a sua linguagem, em muitos aspectos, faz lembrar a do antilhano Aimé Césaire, não só do ponto de vista temático, como pelo recurso estilístico à ressonância verbal."
}

Pensares em Revista, São Gonçalo-RJ, n. 12, p. 160-179, 2018 
dans mes nerfs, il est plus fort que tout, il me faut vivre». Nous remarquons que non seulement le sang noir coule dans les veines de l'auteur ; mais aussi il est la source et le gage de son identité. Ainsi, la dernière strophe montre que Noémia de Sousa s'adonne à un auto-engendrement. Elle prend conscience que c'est le sang noir qui lui a donné la vie ; elle est elle-même l'expression de ce sang indéfectible. Elle lui donne du sens à la fois humainement et esthétiquement, « je vibre » «le sang mêlé que tu m’as légué », « il est à l'origine de ma souffrance, de mon bonheur Mère.».

Comme le souligne Alfredo Bosi dans O ser e o tempo da poesia : « Le poète est celui qui donne du sens ». (1985, p.141. Tradução nossa $\left.{ }^{11}\right)$, dans la mesure où la voix poétique désire donner du sens à sa vie, à son être en étant ce qu'elle est : « je vibre », « il est ma souffrance, mon bonheur Mère ». Le vivre, le souffrir et la symbolique du partage avec sa Mère-Afrique malgré la complexité de la situation coloniale et de la vie. Donc, dans la souffrance, elle s'identifie à sa mère patrie, l'Afrique. La valeur sémantique des verbes cités ci-dessus suggère aussi une fusion organique, un processus d'affirmation de soi. Cela conduit également à une conscience de soi remarquable, à un désir ardent de dire ce qu'on est ou d'être ce qu'on est mozambicaine et africaine.

\section{Langage poétique d'amour à la terre mère et de désaliénation}

L'affirmation de la conscience de soi va se cristalliser davantage dans Sagrada Esperança du poète angolais Agostinho Neto. En cela, il convient d'analyser le poème, «Nous voulons retourner»:

\begin{tabular}{|l|l|}
\hline & Nous devons retourner \\
Havemos de voltar & À nos maisons, à nos labours \\
Às casas, às nossas lavras & Aux plages, à nos champs, \\
às praias, aos nossos campos & nous devons retourner \\
havemos de voltar & À nos terres \\
Às nossas terras & rouges de café, \\
vermelhas do café & à l'éclat du coton blanc \\
brancas de algodão & aux champs de mil reverdissant \\
\hline
\end{tabular}

11 Dans le texte original: "O poeta é doador de sentido."

Pensares em Revista, São Gonçalo-RJ, n. 12, p. 160-179, 2018 


\begin{tabular}{|l|l|}
\hline verdes dos milharais & Nous devons retourner \\
havemos de voltar & À nos mines de diamants, \\
Às nossas minas de diamantes & d'or, de cuivre, de pétrole \\
ouro, cobre, de petróleo & nous devons retourner \\
havemos de voltar & À nos fleuves, nos lacs, nos \\
Aos nossos rios, nossos lagos & montagnes, nos forêts \\
às montanhas, às florestas & nous devons retourner \\
havemos de voltar & À la fraicheur de mulemba, \\
À frescura da mulemba & à nos traditions \\
às nossas tradições & à nos rythmes et au clair de la \\
aos ritmos e às fogueiras & lune \\
havemos de voltar & nous devons retourner \\
À marimba e ao quissange & au marimba et au quissangue \\
ao nosso carnaval & à notre carnaval \\
havemos de voltar & nous devons retourner \\
À bela pátria angolana & à notre belle patrie angolaise \\
nossa terra, nossa mãe & à notre terre, à notre mère \\
havemos de voltar & nous devons retourner \\
À Angola libertada Angola & en Angola libérée, Angola \\
independente & indépendante \\
Cadeia de Aljube, 1960 & Prison d'Aljube, 1960. \\
\hline
\end{tabular}

NETO, 1974, p.127. Tradução nossa.

Ce qui attire l'attention, c'est d'abord le titre du poème. II suggère plus qu'une conscience de soi chez le sujet lyrique. C'est à la fois la manifestation d'un devoir et d'une conviction face aux dérives de l'administration coloniale portugaise. Bien entendu, un devoir et une conviction qui permettent au peuple angolais d'être l'acteur de son destin. En disant « Nous retournons », Agostinho Neto indique implicitement l'urgence d'une libération psychologique et un désir d'affirmation collectif, du fait que son peuple subi les injustices de l'ordre colonial. Dans la première strophe, le poète montre que c'est un devoir de revenir, pour que le peuple angolais puisse jouir de ses « maison, mines, champs ». Au même moment, la valeur anaphorique, « nous retournons » employée tout au long du poème, signifie que c'est aux angolais de construire leur présent et leur avenir. L'emploi du présent de l'indicatif renforce l'idée du devoir collectif - de rejeter la situation coloniale et d'être maître de son destin.

L'imaginaire collectif angolais continue de se mettre en exergue dans la deuxième et la troisième strophe. En cela, l'impératif du retour en Angola symbolise en soi une jouissance de l'esprit, mais aussi du corps. Cela donne une possibilité réelle à l'homme angolais de donner du sens à son existence, puisqu'il peut lui-même profiter de ses, «terres rouge de café », « de coton blanc », « de champs de mil », « de mines Pensares em Revista, São Gonçalo-RJ, n. 12, p. 160-179, 2018 
de diamants, entre autres ». C'est également une façon de retrouver son milieu naturel, comme ses «fleuves », «lacs », « montagnes », « forêts » que le régime colonial portugais a confisqués et exploités.

Dans cette dynamique, la nécessité vitale de retourner à la terre natale s'accentue dans la cinquième et la sixième strophes. II ne s'agit pas d'un retour banal : un retour aux sources africaines. II y a donc une prise de conscience de soi débouchant sur une réappropriation de l'identité angolaise. C'est pourquoi le retour aux sources est une nécessité vitale. Il exige et mérite qu'on se sacrifie pour la Mèrepatrie, l'Angola. En outre, ce retour replace l'Africain dans son contexte culturel authentique, il reprend sa vie naturelle, vivre son africanité. Une telle démarche permet au colonisé d'être à même de reprendre son mode de vie et de vivre sa culture.

En retournant aux sources, aux traditions, le poète incite l'homme angolais à exalter sa culture. A ce propos, l'emploi du /Nous/ dans la structure du poème vient renforcer ce désir collectif, une sorte de volonté collective dans l'affirmation de soi. A travers un langage poétique fortement marqué par des accents patriotiques, Agostinho Neto essaie de retransmettre cette réalité vécue par le peuple angolais. En effet, comme l'affirme Octavio Paz : « le langage indique, représente ; le poème n'explique ni ne représente: il suggère. II ne fait pas allusion à la réalité ; il prétend- et parfois il réussit - à la récréer. La poésie est une plongée, elle s'inspire de la réalité et dès fois elle correspond à la réalité même.» (PAZ, 2003, p.50. Tradução nossa ${ }^{12}$ )

Agostinho Neto nous plonge à travers la poésie dans l'univers angolais et africain. En exaltant le retour aux coutumes, le poète rejette l'assimilation, l'ordre imposé par le régime colonial et désire reprendre son identité africaine. Voilà pourquoi l'aspect rythmé du poème et le choix des mots renvoyant à la réalité africaine comme le «mulemba, marimba, quissangue », servent à exprimer son africanité. On note que le poème est comme un hymne où la voix poétique incarne ou véhicule un message

12 Dans le texte original: "A linguagem indica, representa; o poema não explica nem representa: apresenta. Não alude à realidade; pretende - às vezes o consegue - recriá-la. A poesia é um penetrar, um estar ou ser na realidade."

Pensares em Revista, São Gonçalo-RJ, n. 12, p. 160-179, 2018 
de conscience de soi. En conséquence, les deux dernières strophes évoquent la future patrie angolaise libre d'elle-même et par elle-même.

Le langage poétique d'Agostinho Neto, Néomia de Sousa et José Craveirinha revisite les forces de l'espace socio-culturel de leurs peuples et de l'Afrique durant les affres du colonialisme. C'est ce qui fonde, en dernière instance, leurs langages poétiques de la véritable conscience de soi.

\section{Referências Bibliográficas}

BÂ, A. H. Aspects de la civilisation africaine. Paris : Présence Africaine, 2008 [1972].

BOSI, A. O ser e o tempo da poesia. São Paulo: Cultrix, 1985.

CHABAL, P. Vozes Moçambicanas-literatura e nacionalidade. Lisboa: Veja, 1994.

CRAVEIRINHA, J. Karingana ua Karingana. Lisboa: Caminho, 1990.

Xigubo. Lisboa: Edições 70, 1980.

FERREIRA, M. Literaturas Africanas de Expressão Portuguesa - II. Lisboa: Instituto de Cultura Portuguesa, 1977. (Biblioteca Breve, Vol. 7)

LABAN, M. Moçambique: encontro com escritores Porto: Fundação Eng. António de Almeida, 1998, vol.2.

LEITE, A. M. A Poética de José Craveirinha. 1ª ed. Lisboa: Vega, 1991.

MOMPLE, L. In: FERRO, Ana Maria Mão de (org.), A mulher escritora em África e na América Latina. Évora: Editorial Num, 1999.

NETO, A. Sagrada esperança. Sá de Costa: Lisboa, 1974.

PAZ, O. Signos em Rotação. São Paulo: Perspectiva, 2003.

SERRA C. (org). Identidade, moçambicanidade, moçambicanização. Maputo: Editora Universitária, 1998.

SOUSA, N. de. Sangue negro. Moçambique: Associação dos Escritores de Moçambique, 1988.

\section{Sobre o autor:}

Pensares em Revista, São Gonçalo-RJ, n. 12, p. 160-179, 2018 


\section{Alexandre Coly}

Trabalha como professor de língua, literaturas e culturas lusófonas na Université Assane Seck de Ziguinchor, no Senegal. É pesquisador no CREILAC (Centro de pesquisa interdisplinar em línguas, literaturas, as artes e as culturas- Université Assane Seck de Ziguinchor e pesquisador associado no CELIS - Centro de pesquisas em Literaturas e a Sociopoética -Université- Clermont-Auvergne. Graduado em Letras pela UNESA (2006, RJ), mestre em Literaturas Modernas e Contemporâneas pela Blaise Pascal (2008, França) e doutor (2015) em Estudos lusófonos (Língua, Literaturas e culturas do mundo lusófono) pela Universidade Blaise Pascal e Universidade Cheikh Anta de Dakar, Senegal. É poeta, com dois livros, La natte parlante (2012, Editora Baudelaire) e Le vent des paroles (2015, Editora Edilivre). 